\title{
Simulation of Convex Aspherical Surface Testing with Partial Compensating Interferometer
}

\author{
Yao $\mathrm{Hu}^{1}$, Shaopu Wang ${ }^{1}$, Jingxian Wang ${ }^{1}$, Yan $\mathrm{Ning}^{1}$, Xuemin Cheng ${ }^{2}$ and Qun Hao ${ }^{1, *}$ \\ ${ }^{1}$ Beijing Key Lab. For Precision Optoelectronic Measurement Instrument and Technology, School of Optoelectronics, Beijing \\ Institute of Technology, Beijing, 100081, China \\ ${ }^{2}$ Graduate School at Shenzhen, Tsinghua University, Univ. Town, Xili, Nanshan District, Shenzhen, 518055, China \\ *Corresponding author
}

\begin{abstract}
Large aperture convex aspheric optical components are widely used in imaging systems such as astronomy and space telescopes. Measurement of the figure error of the convex aspherical surface with high precision has been a challenge. We have proposed partial compensating interferometry with catadioptric compensating lens to solve the measurement of convex aspherical surface. In this paper, we dig into the problem of retrace error correction and verify the whole measurement process. We do simulation experiment to measure the wavefront at the exit pupil with digital Moiré phase-shifting technique. And then we do iterative optimization to calculate the figure error and correct the retrace error. The feasibility and accuracy has been demonstrated, which lays the theoretical foundation for the practical measurement.
\end{abstract}

Keywords-convex aspherical surface; catadioptric compensating lens; retrace error; non-null interferometry

\section{INTRODUCTION}

Aspheric optical element has been widely used in astronomy, military and space imaging system to correct aberrations, improve the image quality and simplify the structure of the system, so as to decrease the weight and volume of optical system and decrease the cost. Testing the surface property such as the smooth, shape parameter and figure error is the basis for better fabrication and application of aspherical surfaces. Interferometry is among the most popular measurement techniques for aspherical surfaces.

However, testing convex aspheric surface with interferometry is really a challenge because convex surface can diverge rays, which must be collected and reflected by an optical element, lens or mirror, of sufficient size. Moreover, null testing requires the optical element to generate proper aberration to eliminate the aberration from the tested aspheric and make the output wavefront planar. It will be more difficult to design and fabricate this large-size null optics. Currently computer generated hologram (CGH) on a curved surface [1] or sub-aperture stitching interferometry [2] are adopted for convex aspheric testing. We proposed a partial compensation interferometry [3] to reduce the difficulty in compensation optics design and fabrication, and designed a specific catadioptric compensating lens (PC) [4] to reduce the size of the compensation optics. In this paper, we will do simulations to verify the feasibility of the PC and analyze the accuracy of the method.

\section{MEASUREMENT PRINCIPLE AND METHOdS}

\section{A. Principle of Partial Compensating Interferometry}

We proposed an interferometric method for measuring the surface figure error of asphere based on partial compensating principle and digital moiré phase-shifting technique [3]. Fizeau type interferometer is used for better noise suppression. Partial compensator (PC) is inserted between the reference flat and the measured surface to partially compensating the aberration and make the interference fringes detectable. This is a non-null interferometer. We have two sets of such interferometers. One set $I_{\mathrm{t}}$ is the theoretical interferometer created with optical simulation software Zemax. A series of phase-shifting interferograms are generated with the virtual interferometer in which the measured surface is nominal. Another set is the real interferometer $I_{\mathrm{r}}$ and only one frame of interferogram is captured with camera. Digital Moiré technique is adopted to calculate the wavefront difference $W$ between the theoretical wavefront $W_{\mathrm{t}}$ and the real wavefront $W_{\mathrm{r}}$. Retrace error will rise because of the non-null setting, so $W$ is not equal to twice of the measured figure error $E$. We adopt some optimization algorithms to solve the problem. The details will be discussed in later section.

\section{B. Design of Catadioptric Partial Compensator}

The purpose of designing a $\mathrm{PC}$ is to reduce the aberration of the tested wavefront so that the interference fringes can be recorded and distinguished by the camera. We choose the criterion as the maximum slope of the tested wavefront should be less than $0.45 \lambda /$ pixel [5]. It is determined according to the simulation of the phase-shifting Moire technique and the error brought in by signal processing is controlled to be less than $\lambda / 100$.

The basic structure of the catadioptric PC is displayed in Fig. 1. It consists of a refractive lens $\mathrm{L} 1$ and a spherical mirror M1 with an aperture in the center. The initial configuration of the PC is calculated according to third-order aberration theory. The total spherical aberration of the measured aspheric and the PC should be zero. Then the structure is put into Zemax for optimization. Damp least square method is used with the 
root-mean-square wavefront as the optimization target. The detailed method was proposed in Ref. [4].

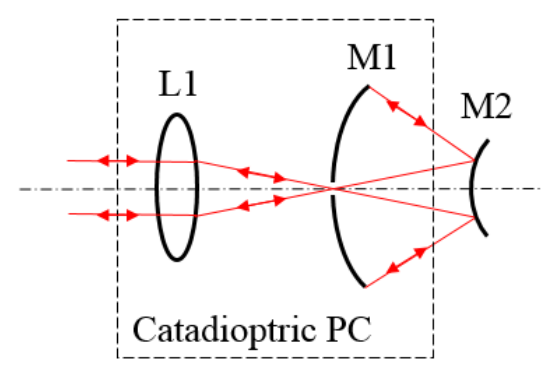

FIGURE I. CONFIGURATION OF THE PC AND MEASURED ASPHERE

\section{Correction of Retrace Error}

As one kind of non-null interferometry, partial compensation method also violate the null condition, resulting in low measurement accuracy. We chose an optimization method similar with that in Ref. [6] to reduce the error.

As we already have a virtual interferometer for calculating the theoretical wavefront $W_{\mathrm{t}}$ and solving the wavefront difference $W$, we can get the real wavefront $W_{\mathrm{r}}=W_{\mathrm{t}}+W . W_{\mathrm{r}}$ is then fitted with annular Zernike polynomials for later processing. We denote the coefficients to be $Z_{\mathrm{r} i}$, where $i=1, \ldots, n$ and $n$ is the number of used Zernike polynomials.

Then we create the second set of virtual interferometer $I_{\mathrm{v}}$ with the same parameter and structure as $I_{t}$, and set the image plane wavefront as the optimization target. The specific merit function is the Zernike coefficients of the image plane wavefront and the target is set exactly as $Z_{\mathrm{r} i}$. The optimization variable is set to be the figure error of the measured surface in the virtual interferometer $I_{\mathrm{v}}$. Specifically, we set the measured surface as a Zernike surface and set the coefficients $Z_{\mathrm{v} i}$ as the variables. Then optimization is run to get a set of $Z_{\mathrm{v} i}$ which makes the image plane wavefront approaching $W_{\mathrm{r}}$. When the error of the image plane wavefront is small enough from $W_{\mathrm{r}}$, we take the corresponding $Z_{\mathrm{v} i}$ and calculate the figure error.

\section{SimUlation AND RESUltS}

\section{A. Systematic Parameters}

We follow the example in Ref. [4]. The cross-section function of the aspheric surface under test can be expressed as

$$
x=\frac{y^{2}}{R_{0}+\sqrt{R_{0}^{2}-\left(1-e^{2}\right) y^{2}}}+E y^{4}+F y^{6}+G y^{8},
$$

(1)

where $x$-axis is along the rotation axis of symmetry and the vertex of the aspheric is taken as the origin, $R_{0}=25.56 \mathrm{~mm},-e^{2}=$ $-1.01, E=3.27 \times 10^{-6}, F=7.72 \times 10^{-10}, G=1.63 \times 10^{-13}$ and the clear aperture of the surface $D_{0}=15.4 \mathrm{~mm}$. The corresponding PC consists of a plano-convex lens made of ZF6 glass and a spherical mirror. The radius of the second surface of the lens is $-209.9 \mathrm{~mm}$ and the central thickness is $8 \mathrm{~mm}$. The radius of the spherical mirror is $47.75 \mathrm{~mm}$. The distance between the last surface of the lens and the spherical mirror is $274.64 \mathrm{~mm}$ and the distance between the mirror and the measured asphere is 38.07 $\mathrm{mm}$.

The real interferometer $I_{\mathrm{r}}$ is also simulated in Zemax. The figure error of the measured asphere is also set with Zernike polynomials. The coefficients of the $8^{\text {th }}, 12^{\text {th }}, 13^{\text {th }}, 16^{\text {th }}$ and $19^{\text {th }}$ polynomials are set to $0.0002 \mathrm{~mm}$ to generate a random figure error for testing simulation. The peak-to-valley (PV) value of the error is $1.27 \lambda$, where the wavelength of the testing system is $632.8 \mathrm{~nm}$.

\section{B. Simulation Results}

The simulated figure error, real interferogram corresponding to $W_{\mathrm{r}}$ (from $I_{\mathrm{r}}$ ) and one of the theoretical phase-shifting interferograms corresponding to $W_{\mathrm{t}}\left(\right.$ from $I_{\mathrm{t}}$ ) are shown in Fig. 2 and Fig. 3. Digital Moiré technique is applied to the real and theoretical interferograms and the four-step Moiré phase-shifting interferograms are shown in Fig. 4. After phase calculation we can get the wavefront difference $W$. Then according to the algorithm proposed above, the figure error is calculated and displayed in Fig. 5. The PV value of the error is $2.22 \lambda$.

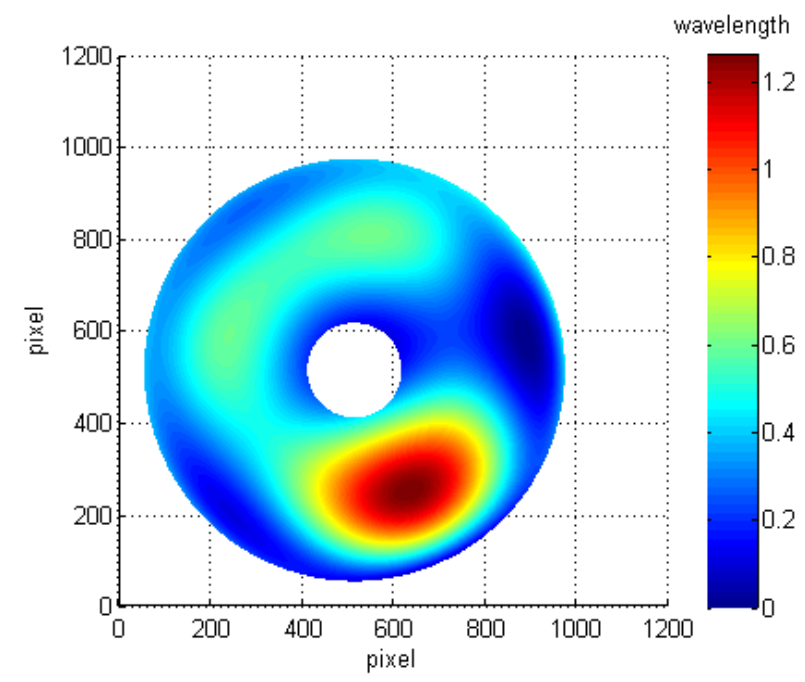

FIGURE II. SIMULATED FIGURE ERROR 


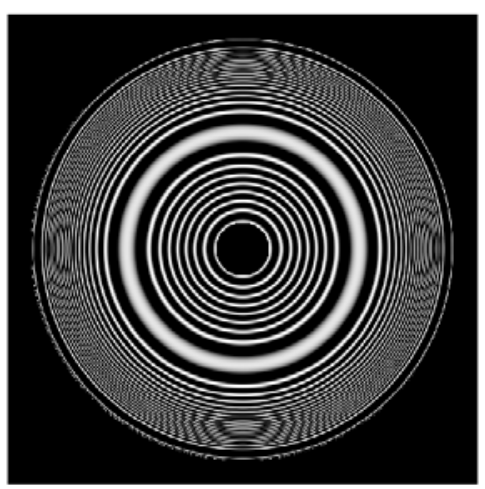

(a)

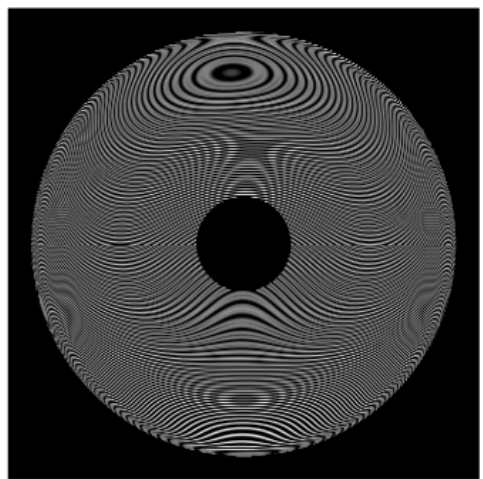

(b)

FIGURE III. SIMULATED REAL AND THEORETICAL INTERFEROGRAMS. (A) REAL INTERFEROGRAM CORRESPONDING TO $W_{\mathrm{R}}$ (FROM $I_{\mathrm{R}}$ ), AND (B) ONE OF THE THEORETICAL PHASE-SHIFTING INTERFEROGRAMS CORRESPONDING TO $W_{\mathrm{T}}\left(\right.$ FROM $\left.I_{\mathrm{T}}\right)$.
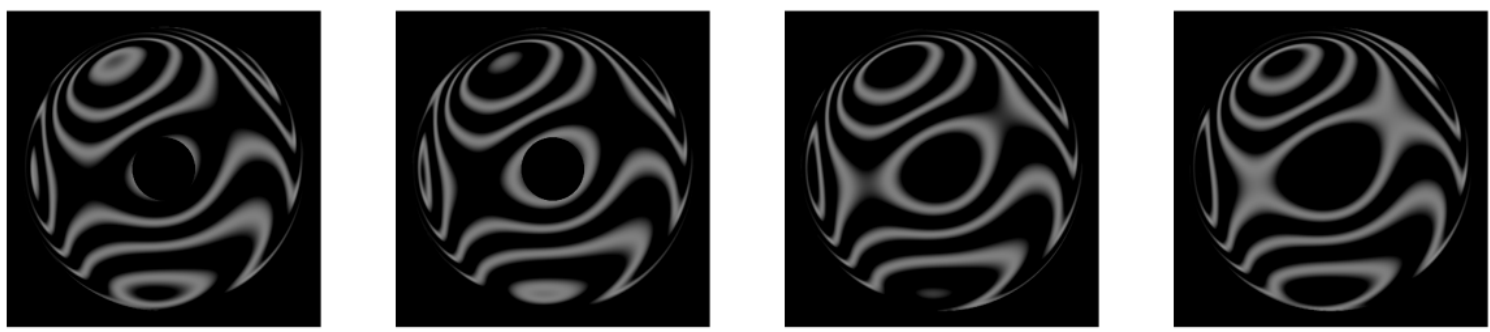

FIGURE IV. FOUR-STEP MOIRÉ PHASE-SHIFTING INTERFEROGRAMS CALCULATED FROM $I_{\mathrm{R}}$ AND $I_{\mathrm{T}}$.

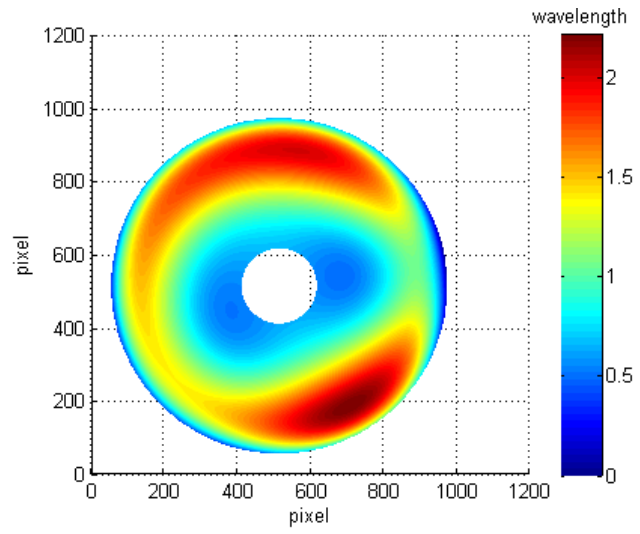

FIGURE V. CALCULATED FIGURE ERROR

\section{Discussion}

The PV value of calculated and simulated figure error differs about $1 \lambda$. However, if we take $95 \%$ aperture of the result, the PV value of the calculated figure error will reduce to about $1.7 \lambda$. The shapes of the two figure error distributions are similar. Possible error sources include wavefront fitting error with annular Zernike polynomials, filtering error during digital Moiré processing and iterative optimization error.

\section{SUMMARY}

In this paper, we did simulation with optical design software Zemax to demonstrating the partial compensation interferometry with catadioptric PC. The result verified the feasibility of the method, but the accuracy is around $0.5 \lambda$ and will be improved in further research.

\section{ACKNOWLEDGEMENT}

This work is supported by National Natural Science Foundation of China No.51327005.

\section{REFERENCES}

[1] H. Liu, Z. Lu, F. Li, and Q. Sun, Design of a novel hologram for full measurement of large and deep convex aspheric surfaces, Opt. Express, 15 (2007) 3120-3126.

[2] S. Chen, C. Zhao, Y. Dai, and S. Li, Stitching algorithm for subaperture 
test of convex aspheres with a test plate, Opt. Laser Technol. 49 (2013) 307-315.

[3] H. Liu, Q. Zhu, Q. Hao, and D. Sha, Design of novel part-compensating lens used in aspheric testing, Proc. SPIE 5253 (2003) 480-484.

[4] J. Wang, Q. Hao, Y. Hu, S. Wang, T. Li, Y. Tian, and L. Li, Convex aspherical surface testing using catadioptric partial compensating system, Proc. AOM2015, Hangzhou (2015).

[5] X. Meng, Q. Hao, Q. Zhu, Y. Hu, Influence of interference fringe's spatial frequency measurement accuracy in digital Moiré phases shifting on the phase interferometry, Chin. J. Lasers 38 (2011) 198-203 (in Chinese).

[6] D. Liu, T. Shi, L. Zhang, Y. Yang, S. Chong, and Y. Shen, Reverse optimization reconstruction of aspheric figure error in a non-null interferometer, Appl. Opt. 53 (2014) 5538-5546. 